\title{
The Choice of Glaucoma Treatment
}

\author{
Silvia Babighian
}

\section{Introduction}

Following detailed and correct diagnosis, the best procedure for the treatment of glaucoma is chosen on the basis of:

- The patient (his/her age, the degree of compliance, his/her life style)

- Glaucoma (the type, the stage of the condition and whether it is associated with other pathologies of the eye).

The therapeutic choice depends on the stage of the condition in cases of open-angle glaucoma. In the initial phases, when the optic nerve and the visual field have still been preserved, the surgeon should prescribe drug therapy. If the patient is not adequately compensated, not compliant or is allergic or intolerant to the drugs, a selective trabeculoplasty is recommended; this option is more conservative and associated with fewer side effects compared to the older argon laser trabeculoplasty technique. In the advanced phases, when there has been damage to the optic disc and the visual field that may be progressing, surgery is the best option. One of the techniques available is canaloplasty, a technique that was introduced fairly recently. It is associated with low levels of trauma and the absence of a sub-conjunctival bleb means that the success is not conditioned by conjunctiva fibrosis. Moreover, this technique is an excellent therapeutic option due to the low percentage of complications and the good results reported in the literature in the medium-term. Nevertheless, the long learning curve, the need for dedicated instruments and the fact that it is not always possible to incannulate the Schlemm Canal, induce many surgeons to opt for Cairns trabeculectomy; this is still considered to be the most

S. Babighian, MD, $\mathrm{PhD}$

Head of Glaucoma Service, Ospedale Sant'Antonio, Padova, Italy

e-mail: s.babighian@gmail.com efficacious surgical approach to achieve the target pressure to arrest the damage induced by the glaucoma. Both these glaucoma procedures can be combined with cataract extraction if present. In the terminal phases or in the event of failure of the standard surgical technique, if there is residual visus, the surgeon can opt to insert a drainage implant. In the event of very poor or absent visual acuity, particularly if associated with eye-bulb pain caused by ocular hypertension, the surgeon may use a diode laser to perform cyclophotocoagulation of the ciliary body, a procedure that has recently been replaced by cycloablation of the ciliary body using ultrasound. In the case of closed-angle glaucoma in a young patient or in the initial phases of the disease, the situation can be resolved using local drug therapy (miotics, in particular) and YAG laser iridotomy to resolve the pupil block and avoid any acute episodes. In the full-blown condition of the disease, the surgeon must consider a filtering surgical technique if goniosynecchias are present; alternately, he may opt for the surgical extract of the cataract using phacoemulsification, or the extracapsular extraction if there has been appositional angle closure. In the case of congenital glaucoma, the surgical approach is the only valid therapeutic option. If the cornea is transparent, the surgeon can perform a goniotomy (or trabeculotomy) procedure; on the contrary, if the cornea is cloudy, trabeculectomy can be performed, or he can opt for the trabeculectomy. In secondary glaucoma, surgery is the option associated with greatest success. Frequently, it will be associated with laser techniques that may contribute to increasing the success of achieving the target intraocular pressure. In conclusion, each glaucoma treatment must be adapted to the type of patient and to the type of glaucoma. The surgeon must carefully explain to the patient the reasons behind the therapeutic decisions, explaining the risks, the possible complications and the impact these choices will have on the quality of life; he must also explain that if no adequate treatment is available, there may be irreversible damage to the visual function that might even result in blindness. 